\title{
A New Method for Robust Damping and Tracking Control of Scanning Probe Microscope Positioning Stages
}

\author{
Andrew J. Fleming, Member, IEEE, Sumeet S. Aphale, Member, IEEE, \\ and S. O. Reza Moheimani, Senior Member, IEEE
}

\begin{abstract}
This paper demonstrates a simple second-order controller that eliminates scan-induced oscillation and provides integral tracking action. The controller can be retrofitted to any scanning probe microscope with position sensors by implementing a simple digital controller or operational amplifier circuit. The controller is demonstrated to improve the tracking bandwidth of an NT-MDT scanning probe microscope from $15 \mathrm{~Hz}$ (with an integral controller) to $490 \mathrm{~Hz}$ while simultaneously improving gainmargin from 2 to $7 \mathrm{~dB}$. The penalty on sensor induced positioning noise is minimal. A unique benefit of the proposed control scheme is the performance and stability robustness with respect to variations in resonance frequency. This is demonstrated experimentally by a change in resonance frequency from 934 to $140 \mathrm{~Hz}$. This change does not compromise stability or significantly degrade performance. For the scanning probe microscope considered in this paper, the noise is marginally increased from 0.30 to $0.39 \mathrm{~nm}$ rms. Open- and closed-loop experimental images of a calibration standard are reported at speeds of 1,10 , and 31 lines per second (with a scanner resonance frequency of $290 \mathrm{~Hz}$ ). Compared with traditional integral controllers, the proposed controller provides a bandwidth improvement of greater than 10 times. This allows faster imaging and less tracking lag at low speeds.
\end{abstract}

Index Terms-Feedback control, high-speed scanning, resonance damping, scanning probe microscopy, tracking.

\section{INTRODUCTION}

$\mathbf{T}$ O INVESTIGATE matter at nanometer and subnanometer scales, scanning probe microscopy (SPM) was introduced more than two decades ago [1], [2]. A key component of these instruments is the nanopositioning stage used to scan or position the probe or sample. Many nanopositioning device geometries have been proposed and tested for this purpose [3]-[7]. However, due to the mechanical simplicity and large scan range, piezoelectric tube scanners have become the most popular devices used in commercial SPM systems [8].

Manuscript received February 16, 2009; revised May 17, 2009; accepted September 1, 2009. Date of publication September 29, 2009; date of current version July 9, 2010. This work was supported by the Australian Research Council Center of Excellence for Complex Dynamic Systems and Control, and by the Australian Research Council Discovery Project DP0666620. The review of this paper was arranged by Associate Editor E. T. Yu.

A. J. Fleming and S. O. R. Moheimani are with the School of Electrical Engineering and Computer Science, University of Newcastle, Callaghan, N.S.W. 2308, Australia (e-mail: andrew.fleming@newcastle.edu.au).

S. S. Aphale is with the Center for Applied Dynamics Research, School of Engineering, Kings College, University of Aberdeen, Aberdeen AB24 3FX, U.K.

Color versions of one or more of the figures in this paper are available online at http://ieeexplore.ieee.org.

Digital Object Identifier 10.1109/TNANO.2009.2032418
These tube scanners have two inherent problems that degrade the positioning performance of the scanner, viz: 1) resonant modes due to the mechanical construction [9], [10]; and 2) nonlinear behavior due to hysteresis and creep in the piezoelectric material [11], [12].

Piezoelectric tube scanners feature a dominant, lightly damped, low-frequency resonant mode in their frequency response. High-frequency components of the reference input and/or exogenous noise can excite this resonant mode causing erroneous vibration and large positioning errors. In most piezoelectric tubes applications, the fastest possible open-loop scan frequency is limited to less than $1 \%$ of the resonance frequency. Though the frequency of this resonant mode depends on the physical dimensions of the tube scanner, typical resonance frequencies are less than $1 \mathrm{kHz}$. Thus, the fastest achievable scans are at speeds of less than $10 \mathrm{~Hz}$. This speed constraint is further restricted by the presence of piezoelectric nonlinear effects such as hysteresis and creep. These nonlinearities necessiate the use of closed-loop tracking controllers such as integral controllers. Detrimentally, controllers with integral action are severely limited in bandwidth by the mechanical resonance that imposes a low gain-margin. Contrary to the low speed achievable with piezoelectric tube scanners, many scanning applications are demanding faster scan rates with greater accuracy and resolution, [7], [13]-[17].

To improve the gain-margin and closed-loop bandwidth of nanopositioning systems, notch filters or inversion filters can be employed. These techniques are popular as they are simple to implement and can provide excellent closed-loop bandwidth, up to or greater than the resonance frequency [18]. The major disadvantage is the requirement for an accurate system model. If the system resonance frequency shifts by only $10 \%$, a high-gain inversion-based feedback controller can become unstable. In most applications, this is unacceptable as the load mass, and hence resonance frequency of a nanopositioner can vary significantly during service. As a result of this sensitivity, high-performance inversion-based controllers are only applied in niche applications where the resonance frequency is stable, or when the feedback controller can be continually recalibrated [18].

To reduce errors resulting from the system resonance, various closed-loop damping techniques have been proposed. Positive position feedback control and polynomial-based controller designs have been shown to adequately damp the resonant mode (see [19], [20]). The application of active and passive 


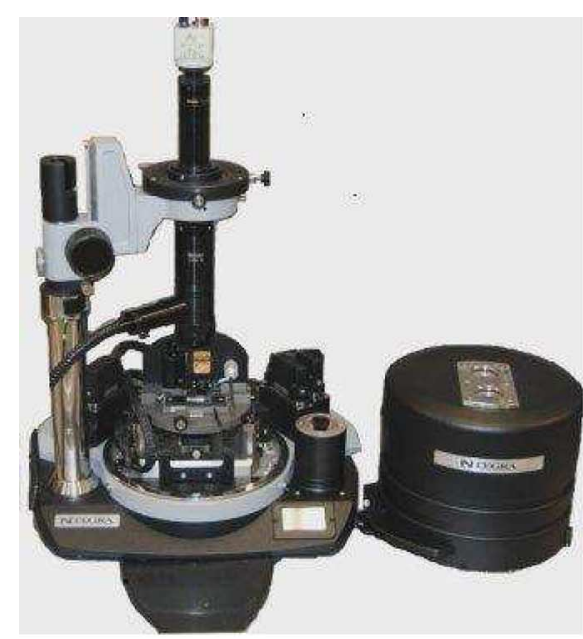

(a)

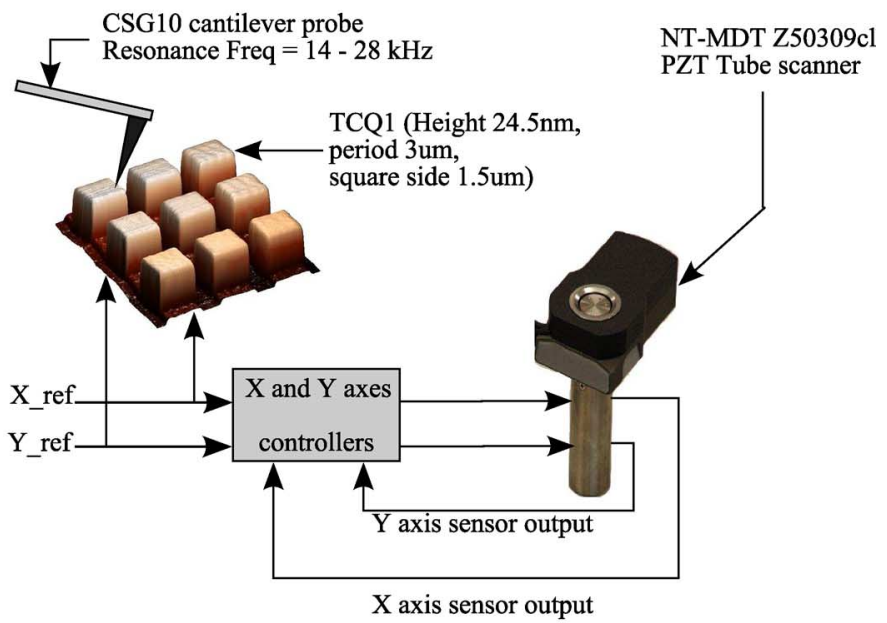

(b)

Fig. 1. (a) NT-MDT Ntegra scanning probe microscope. (b) Experimental scanner configuration.

shunt damping techniques for piezoelectric tube scanners was reported in [21] and [22]. Other active damping techniques, including receding horizon control, have also been proposed (see [23], [24]). For a detailed overview on this topic, the reader is referred to [25].

In addition to feedback control, feedforward or inversion based control had been proposed for both open- and closed-loop nanopositioning systems [25], [26]. Good reference tracking can be achieved if the plant model or its frequency response are known with high accuracy. The foremost difficulty with inversion-based control is the lack of robustness to variations in plant dynamics, especially if the system is resonant [26], [27]. However, this problem only exists with static feedforward controllers. More recently, iterative techniques have been reported that eliminate both vibration and nonlinearity in systems with periodic inputs [28]. Although such techniques originally required a reference model [28], in 2008, both Kim and Zou [29] and Li and Bechhoefer [30] presented techniques that operate without any prior system knowledge. Both techniques achieve essentially perfect tracking regardless of nonlinearity or dynamics.

The disadvantages associated with iterative feedforward techniques [29], [30] are the implementation complexity, insensitivity to external disturbance, and requirement for periodic signals. As both methods operate in the frequency domain, a single iteration requires a number of input and output periods and the computation of Fourier and inverse Fourier transforms. Even considering the signal processing capabilities available in modern scanning probe microscopes, the required computations are significant. Imaging experiments using these techniques are yet to be demonstrated. The requirement for periodic signals also precludes imaging modes such as spectroscopy and surface modification.

Recently, the integral resonant control (IRC) scheme was demonstrated as a simple means for damping multiple resonance modes of a cantilever beam [31]. The IRC scheme employs a constant feedthrough term and a simple first-order controller to achieve substantial damping of multiple resonance modes. This technique was applied directly to a piezoelectric tube scanner in [32]. However, direct application provides only vibration control, it does not result in a zero steady-state error, or elimination of drift and nonlinearity at low frequencies.

\section{A. Contribution of This Paper}

In this paper, a standard regulator controller ${ }^{1}$ is derived from the integral resonant control scheme. The regulator turns out to be a first-order low-pass filter and is also straight-forward to implement. A major benefit of the regulator form is that it can be enclosed in a simple tracking control loop to eliminate drift and effectively reduce nonlinearity at low frequencies.

Due to the implementation simplicity, damping performance and excellent robustness properties of the proposed controller, it is an excellent alternative to the standard proportional-integral (PI) control algorithms presently used in many commercial SPMs.

In this paper, we demonstrate an IRC damping controller with integral tracking action applied to an NT-MDT Ntegra SPM. Experimental results show greater than ten times improvement in tracking bandwidth with improved stability margins and disturbance rejection. This allows the microscope to operate at speeds exceeding 30 lines/s with no mechanical modifications.

This paper is organized as follows. Section II describes the experimental setup. Details of the control design are then given in Section III. The controller is then implemented in Section IV. Open- and closed-loop scan results are also compared in Section IV. The noise performance is evaluated in Section V followed by details on analog circuit implementation in Section VI. Conclusions are drawn in Section VII.

\section{EXPERIMENTAL SETUP}

An NT-MDT Ntegra SPM was used to implement and test the proposed control strategy. A signal access module allows

\footnotetext{
${ }^{1} \mathrm{~A}$ regulator controller appears between the error summation and the plant.
} 


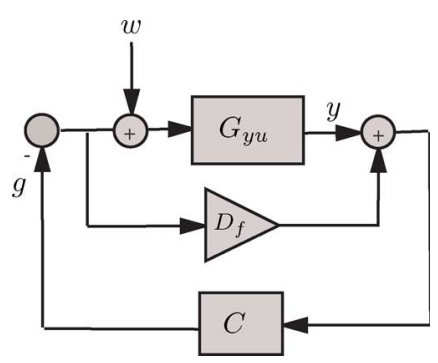

Fig. 2. Integral resonant control scheme [31].

direct access to the scanner electrodes and reference trajectory. The scanner is an NT-MDT Z50309cl piezoelectric tube scanner with $100 \mu \mathrm{m}$ range. The tube scanner has quartered internal and external electrodes allowing the scanner to be driven in a bridged configuration. That is, the internal and external electrodes are driven with equal but opposite voltages. Capacitive sensors are used to measure the resulting displacement in each axis with a sensitivity of $0.158 \mathrm{~V}$ per micrometer.

For modeling purposes, the scanner is treated as a two-input two-output system. The two inputs are the voltages applied to the $x$ - and $y$-axis amplifiers while the outputs are the corresponding capacitive sensor voltages. All of the frequency responses were recorded with an HP-35670A Spectrum Analyzer. The control strategy was implemented using a dSPACE-1103 rapid prototyping system.

\section{CONTROL DESIGN}

The foremost control objective in nanopositioning is to minimize tracking error. As the system is nonlinear, this requires integral action in the control loop. For high-speed operation the closed-loop system must be inverted either offline or with a feedforward controller. Although this is straight-forward to accomplish, the resulting performance can be highly sensitive to small changes in resonance frequency. In this paper, a damping controller is utilized to attenuate the system's first resonant mode. This provides improved bandwidth without the need for accurate plant models or inversion. The damping controller is highly robust to changes in resonance frequency and also provides improved disturbance rejection.

A model of the system described in Section II was procured using the frequency domain subspace technique [33]. In the next sections, this model is described as $G_{y u}$ and has the following parameters:

$$
G_{y u}=\frac{0.04976 s^{2}+26.84 s+1.746 e 006}{s^{2}+43.6 s+3.32 e 006} .
$$

\section{A. Damping Controller}

As discussed in Section I, IRC was introduced as a means for augmenting the structural damping of resonant systems with collocated sensors and actuators. A diagram of an IRC loop is shown in Fig. 2. It consists of the collocated system $G_{y u}$, an artificial feedthrough $D_{f}$, and a controller $C$. The input disturbance $w$ represents environmental disturbance but can also be used to obtain some qualitative information about the closed-loop re-
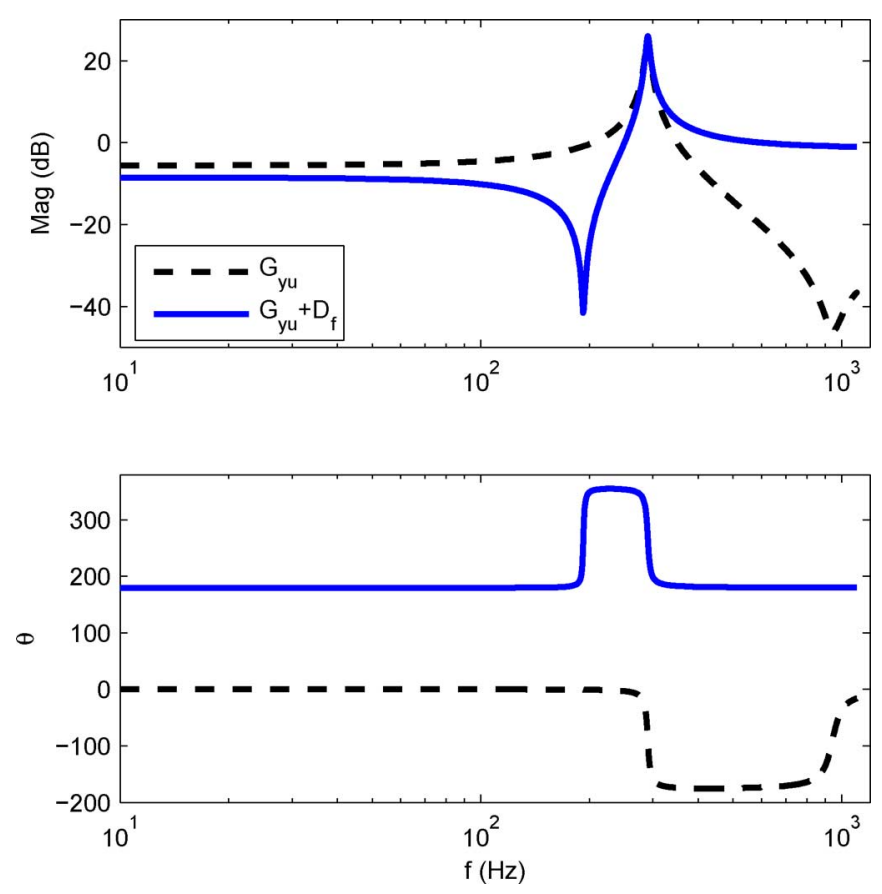

Fig. 3. Frequency response from the applied $x$-axis voltage to the measured sensor voltage in the same axis $G_{y u}$. The system with artificial feedthrough is also shown $G_{y u}+D_{f}$, where $D_{f}=-0.9$. The $180^{\circ}$ phase change of $G_{y u}+D_{f}$ is due to the negative feedthrough that also makes the system inverting.

sponse to piezoelectric nonlinearity. That is, if the disturbance rejection at the scan frequency and first few harmonics is large, a significant reduction in hysteresis could be expected.

The first step in designing an IRC controller is to select, and add, an artificial feedthrough term $D_{f}$ to the original plant $G_{y u}$. It has been shown that a sufficiently large and negative feedthrough term will introduce a pair of zeros below the first resonance mode and also guarantee zero-pole interlacing for higher frequency modes [31]. This new system is referred to as $G_{y u}+D_{f}$. For a detailed explanation regarding the choice of a suitable feedthrough term, the reader is referred to Theorem 2 in [31].

For the model $G_{y u}$ described in (1), a feedthrough term of $D_{f}=-0.9$ is sufficient to introduce a pair of zeros below the first resonance mode. The frequency responses of the openloop system $G_{y u}$ and the modified transfer function $G_{y u}+D_{f}$, where $D_{f}=-0.9$, are plotted in Fig. 3. Note the change from a pole-zero pattern to a zero-pole pattern.

The key behind the IRC is the phase response of $G_{y u}+D_{f}$, which now lies between 180 and $360^{\circ}$ as shown in Fig. 3. As the higher order modes are guaranteed to exhibit a zero-pole ordering, the phase response does not exceed this range.

Due to the bounded phase of $G_{y u}+D_{f}$ a simple negative integral controller

$$
C=\frac{-k}{s}
$$

can be applied directly to the system. To examine the stability of such a controller, we consider the loop-gain $C\left(G_{y u}+D_{f}\right)$. For stability, the phase of the loop-gain must be within $\pm 180^{\circ}$ 


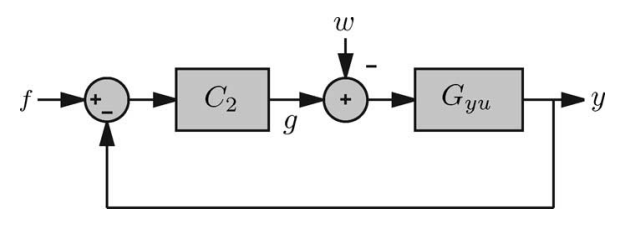

Fig. 4. The integral resonant controller of Fig. 2 rearranged in regulator form.

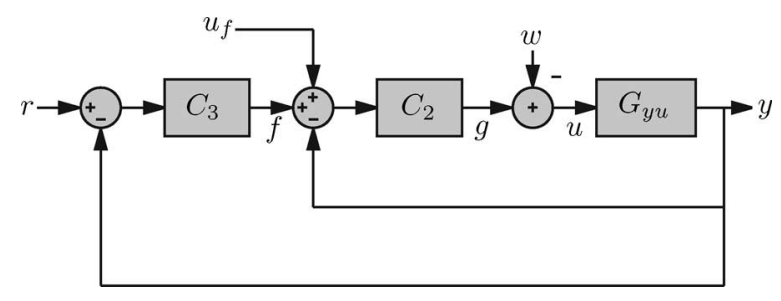

Fig. 5. Tracking control system with the damping controller $C_{2}(s)$ and tracking controller $C_{3}(s)$. The feedforward input $u_{f}$ is discussed in Section III-C.

while the gain is greater than zero. The phase of the loop-gain $C\left(G_{y u}+D_{f}\right)$ is equal to the phase of $G_{y u}+D_{f}$ minus $180^{\circ}$ for the negative controller gain and a further $90^{\circ}$ for the single controller pole. The resulting phase response of the loop-gain lies between $+90^{\circ}$ and $-90^{\circ}$. That is, regardless of controller gain, the closed-loop system has a phase margin of $90^{\circ}$ and an infinite gain-margin with respect to $G_{y u}+D_{f}$.

A suitable controller gain $k$ can easily be selected to maximize damping using the root-locus technique [31].

\section{B. Tracking Controller}

After implementing an IRC controller, shown in Fig. 2, a secondary integral control loop cannot be directly closed around the output of $G_{y u}$. The feedthrough term $D_{f}$ and the location of the summing junction prevent the possibility for integral action.

To incorporate an additional control loop, the feedback diagram must be rearranged so that an additional input does not appear as a disturbance. This can be achieved by finding an equivalent regulator that provides the same loop gain but with an input suitable for tracking control. In Fig. 2, the control input $g$ is related to the measured output $y$ by

$$
g=C\left(y-D_{f} g\right)
$$

thus, the equivalent regulator $C_{2}$ is

$$
C_{2}=\frac{C}{1+C D_{f}} .
$$

When $C=-k / s$, the equivalent regulator is

$$
C_{2}=\frac{-k}{s-k D_{f}} .
$$

A diagram of the equivalent regulator loop formed by $C_{2}$ and $G$ is shown in Fig. 4. This loop is easily enclosed in a secondary outer loop to achieve integral tracking. A control diagram of this configuration is shown in Fig. 5. Due to the inverting behavior of the IRC loop, the tracking controller $C_{3}$ is a negative integral controller

$$
C_{3}=\frac{-k_{i}}{s}
$$

The transfer function of the closed-loop system is

$$
\frac{y}{r}=\frac{C_{2} C_{3} G_{y u}}{1+C_{2}\left(1+C_{3}\right) G_{y u}} .
$$

In addition to the closed-loop response, the transfer function from disturbance to the regulated variable $y$ is also of importance. This can be found as

$$
\frac{y}{w}=\frac{G_{y u}}{1+C_{2}\left(1+C_{3}\right) G_{y u}} .
$$

That is, the disturbance input is regulated by the equivalent controller $C_{2}\left(1+C_{3}\right)$.

\section{Feedforward Input}

Feedforward inputs can be used to improve the bandwidth of a closed-loop system by bypassing the tracking controller or inverting dynamics [26], [34], [35]. Inversion-based feedforward provides the best performance but is also sensitive to modeling inaccuracies and system variations during service. Here, where a change in resonance frequency from 260 to $900 \mathrm{~Hz}$ is considered, inversion-based feedforward cannot be applied. Such wide variations in resonance frequency would result in unacceptable modeling error and detrimental feedforward performance [27]. However, simply using the inverse dc gain of the system provides some improvement in tracking lag and is beneficial in this application.

In Fig. 5, the feedforward input is denoted $u_{f}$. This signal is generated from the reference input and the dc gain of the damped system, i.e.,

$$
u_{f}=r\left(\left.\frac{C_{2} G_{y u}}{1+C_{2} G_{y u}}\right|_{s=0}\right)^{-1} .
$$

\section{EXPERIMENTAL IMPLEMENTATION}

\section{A. Controller Design}

In this section, the proposed control scheme is implemented on the atomic force microscope (AFM) discussed in Section IV. For the sake of comparison, three controllers were considered: 1) an integral tracking controller; 2) an integral tracking controller with feedforward; and 3) an integral tracking controller with IRC damping and a feedforward input. Diagrams of the three control strategies are given in Fig. 6. The design and performance of each controller is discussed in the following. A summary of the controllers is contained in Table I.

1) Integral Tracking Controller: The integral controller was designed to maximize tracking bandwidth. The maximum gain was restricted to $k_{i}=80$ by the gain-margin of only $2.5 \mathrm{~dB}$. The low gain-margin is due to the lightly damped resonance mode at $575 \mathrm{~Hz}$. As the resonance has a sharp-phase response at a frequency much higher than the controller's crossover frequency, the system phase margin is dominated by the integral controller and remains at $90^{\circ}$. The experimental frequency response, showing a $15 \mathrm{~Hz}$ bandwidth, and time-domain response to a $10 \mathrm{~Hz}$ triangular scan is shown in Fig. 6. 

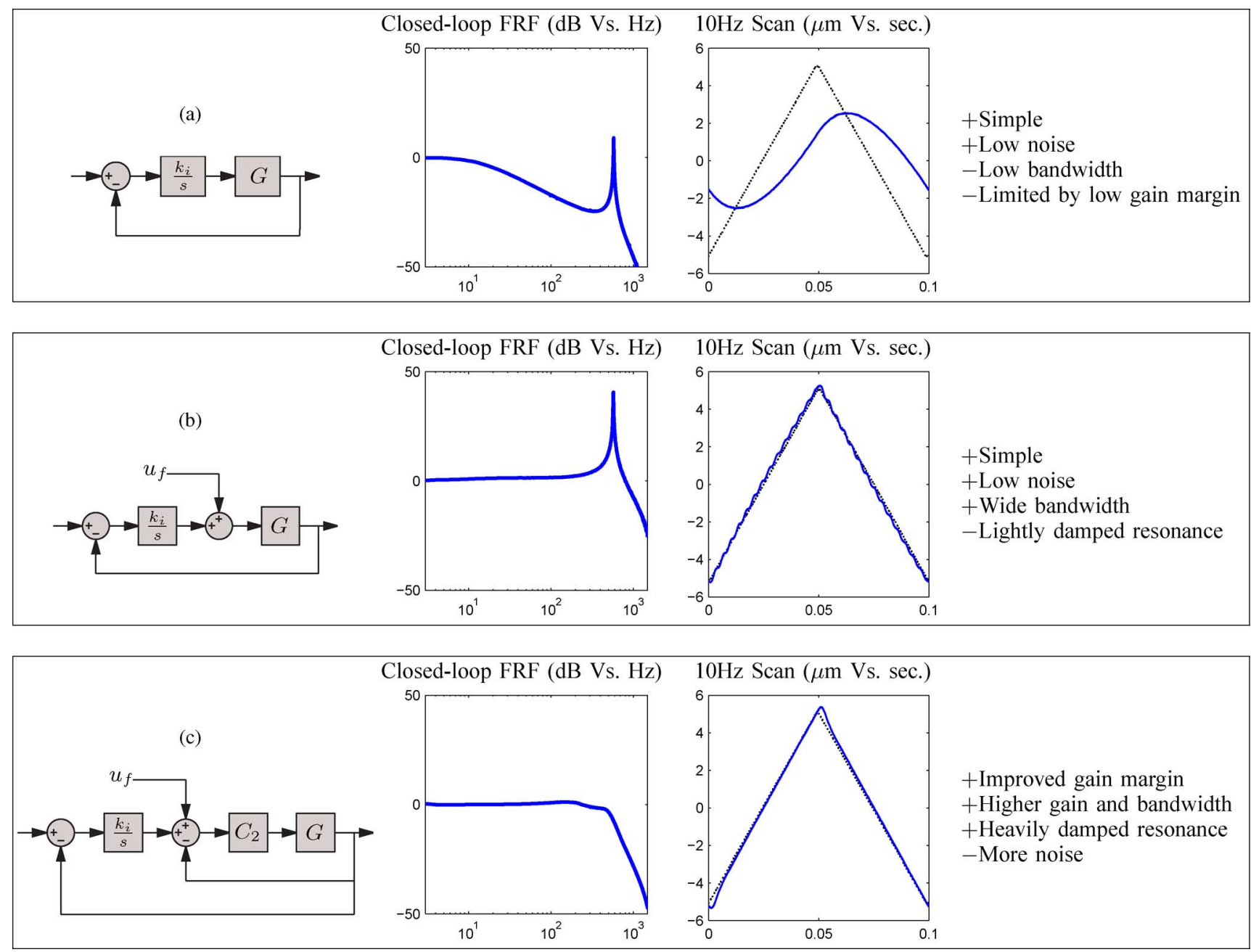

Fig. 6. Comparison of control strategies from simplest to more complicated. The frequency responses are measured from the applied reference to the measured sensor voltage. (a) Integral control. (b) Integral + feedforward. (c) Integral + IRC + feedforward.

TABLE 1

SUMMARY OF IMPLEMENTED CONTROLLERS AND RESULTING CLOSED-LOOP BANDWIDTH

\begin{tabular}{|c|c|c|c|c|}
\hline Controller & $C_{3}$ & $C_{2}$ & $u_{f}$ & Bandwidth \\
\hline \hline Integral & $\frac{(80)}{s}$ & 0 & 0 & $15 \mathrm{~Hz}$ \\
\hline Integral + FF & $\frac{(80)}{s}$ & 0 & 1.88 & $251 \mathrm{~Hz}$ \\
\hline Integral + IRC + FF & $\frac{-(400)}{s}$ & $\frac{-(1800)}{s-(1800)(-0.9)}$ & 0.91 & $490 \mathrm{~Hz}$ \\
\hline
\end{tabular}

2) Integral Controller With Feedforward: By adding a feedforward input to the integral controller, as shown in Fig. 6, the bandwidth can be extended to $251 \mathrm{~Hz}$. However, the majority of this bandwidth is uncontrolled and the open-loop dynamics now appear in the tracking response. The time-domain response exhibits significant oscillation which is highly undesirable in microscopy applications.

3) Integral Controller With IRC Damping and Feedforward: Following the procedure in Section III-B an IRC damping controller was first designed for the system. From a root-locus plot, the maximum damping was found to occur at $k=1800$. An integral controller was then designed for the damped system. With a gain of $k_{i}=400$ the resulting closed-loop system has a band- width of $490 \mathrm{~Hz}$ while maintaining a $7 \mathrm{~dB}$ gain-margin and $50^{\circ}$ phase margin. This is a vast improvement in both bandwidth and stability margins compared to the controller in Section IV-A1.

While the control design has only been discussed for the $x$ axis, an identical controller was designed for the $y$-axis. With both controllers present, the frequency response of each axis and the corresponding cross coupling is plotted in Fig. 7. An important observation is that the resonance in both cross-coupling transfer functions has been significantly damped. This guarantees that fast motion in one axis will not induce large oscillations in the adjacent axis, a highly desirable characteristic. It should also be noted that nominal cross-coupling magnitude is low $(-40 \mathrm{~dB})$. This implies that the $x$ and $y$ axes are effectively decoupled and can be treated independently as two single-input single-output (SISO) loops.

\section{B. Imaging Performance}

In this section, experimental images are presented that demonstrate the effectiveness of the IRC controller discussed in the previous subsection. A comparison with open-loop performance is also included. The open-loop results illustrate 

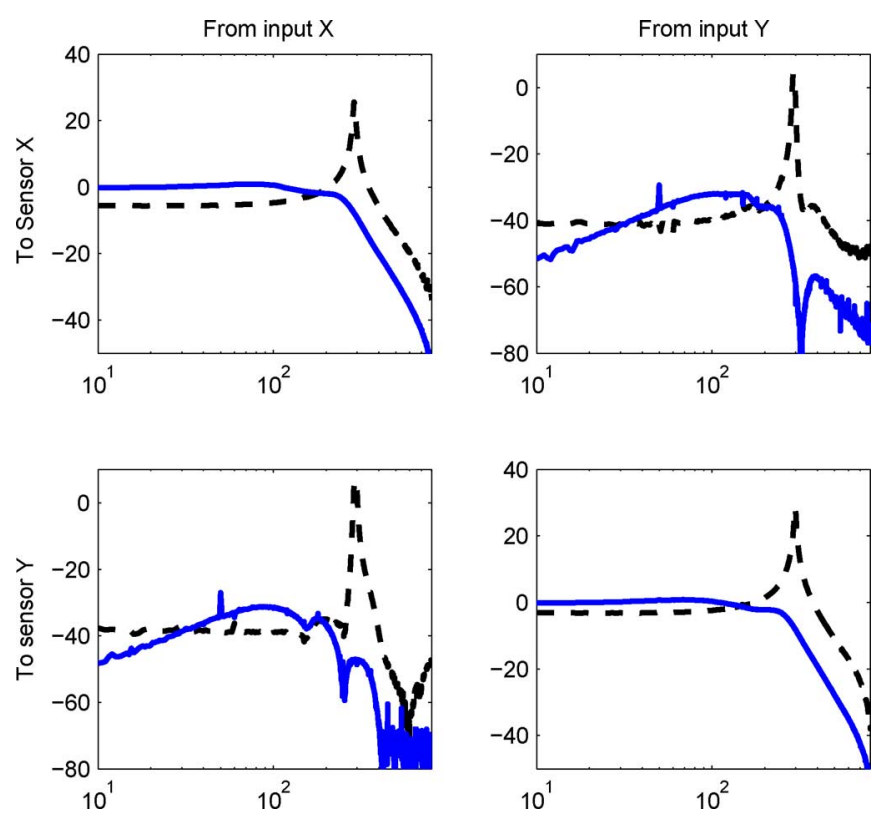

Fig. 7. Multivariable magnitude frequency response plotted (in decibel versus hertz). The dashed and solid lines are the open- and closed-loop responses, respectively.

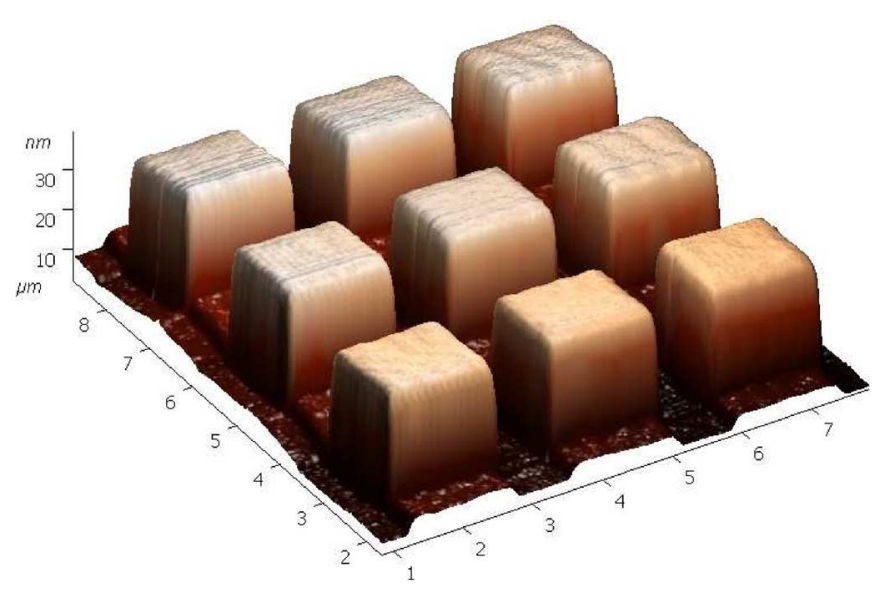

Fig. 8. MikroMasch TGQ1 calibration grating. The feature height is $24.5 \mathrm{~nm}$ with $3 \mu \mathrm{m}$ period. This image was obtained using constant-force contact mode with a $1 \mathrm{~Hz}$ line rate.

the imaging artefacts that arise from scan-induced vibration, and also closely resemble the images obtained using the microscope's built-in controller. The built-in controller is an integral plus feedforward controller as discussed in Section IV-A2. At the frequencies considered, the integral part of the controller is negligible, and the system operates effectively in open-loop.

The sample under consideration is a MikroMasch TCQ1 grating with a feature height of $24.5 \mathrm{~nm}$ and period of $3 \mu \mathrm{m}$. Depicted in Fig. 8, this grating is useful for quantifying oscillation and nonlinearity in both axes simultaneously. All of the following images were recorded in constant-height contact mode with a NT-MDT CSG10 cantilever with a resonance frequency of $20 \mathrm{kHz}$ and stiffness of $0.1 \mathrm{~N} / \mathrm{m}$.

Images of the grating were recorded in open- and closed-loop at 1,10 , and 31 lines/s. At $1 \mathrm{~Hz}$, there is no distinguishable difference between open- and closed-loop control and these images are not included. In Fig. 9, the oscillation in the open-loop $10 \mathrm{~Hz}$ scan is clearly visible in both the image and measured $x$ axis displacement. With the controller activated, the oscillation and corresponding artifacts are eliminated.

At $31 \mathrm{~Hz}$ line rate, the induced oscillation again severely degrades image quality. Although the magnitude of oscillation is greater than the $10 \mathrm{~Hz}$ scan, the image does not appear significantly more distorted as the period of oscillation is similar to the period of the sample. With closed-loop control, the oscillation is again eliminated. However, the overshoot and tracking-lag of the system now causes significant distortion over approximately one third of the scan range. This is due to the high scan rate relative to the bandwidth of the system. At $31 \mathrm{~Hz}$, only the first five harmonics of the input triangle signal appear below the resonance frequency. Overshoot can be reduced by removing the feedforward input or by using a different feedforward architecture, but at the expense of increased tracking lag [26]. In this paper, as a time delay to account for tracking lag cannot be incorporated into the microscope controller, it is desirable to minimize tracking lag at the expense of overshoot.

At higher scan rates where overshoot and tracking lag become significant, the performance can be improved by model based inversion [35] but at the expense of robustness [27]. As this paper aims to provide good performance over an extremely wide range of operating conditions, feedforward inversion is not considered beneficial. Performance improvements can also be achieved by shaping the input triangle signal to remove energy above the fifth harmonic. A review of techniques for achieving this and a method for generating optimal input signals is contained in [36]. These techniques are not used here, as they require modification of the microscope control logic, and are thus not immediately straight forward to implement, which is a requisite in this paper.

\section{Performance Robustness}

During service, the sample mass and resonance frequency of SPM scanners can vary widely. The highest resonance frequency occurs while the scanner is unloaded, this can drop by $80 \%$ as additional mass such as liquid cells and heating elements are added. Such large variations in resonance frequency are not often discussed in the literature, as it can be extremely difficult to design controllers that are even stable, let alone provide reasonable performance, over such ranges. However, to be of practical value to SPM users and designers, this issue is of primary concern.

A benefit of the control technique discussed in Section III-A is the high robustness to changes in resonance frequency with respect to both stability and performance. This is a unique characteristic that is ideal for SPM scanner control. For the microscope described in Section II, the resonance frequency is $934 \mathrm{~Hz}$ when unloaded. With a sample holder and heating element, this reduces to $290 \mathrm{~Hz}$. A further reduction to $140 \mathrm{~Hz}$ is possible if additional mass such as a liquid cell or magnetic coil is added. The open-loop frequency response under these conditions is plotted in Fig. 10. The closed-loop response is also shown. In all cases, the controller remains stable and provides good 

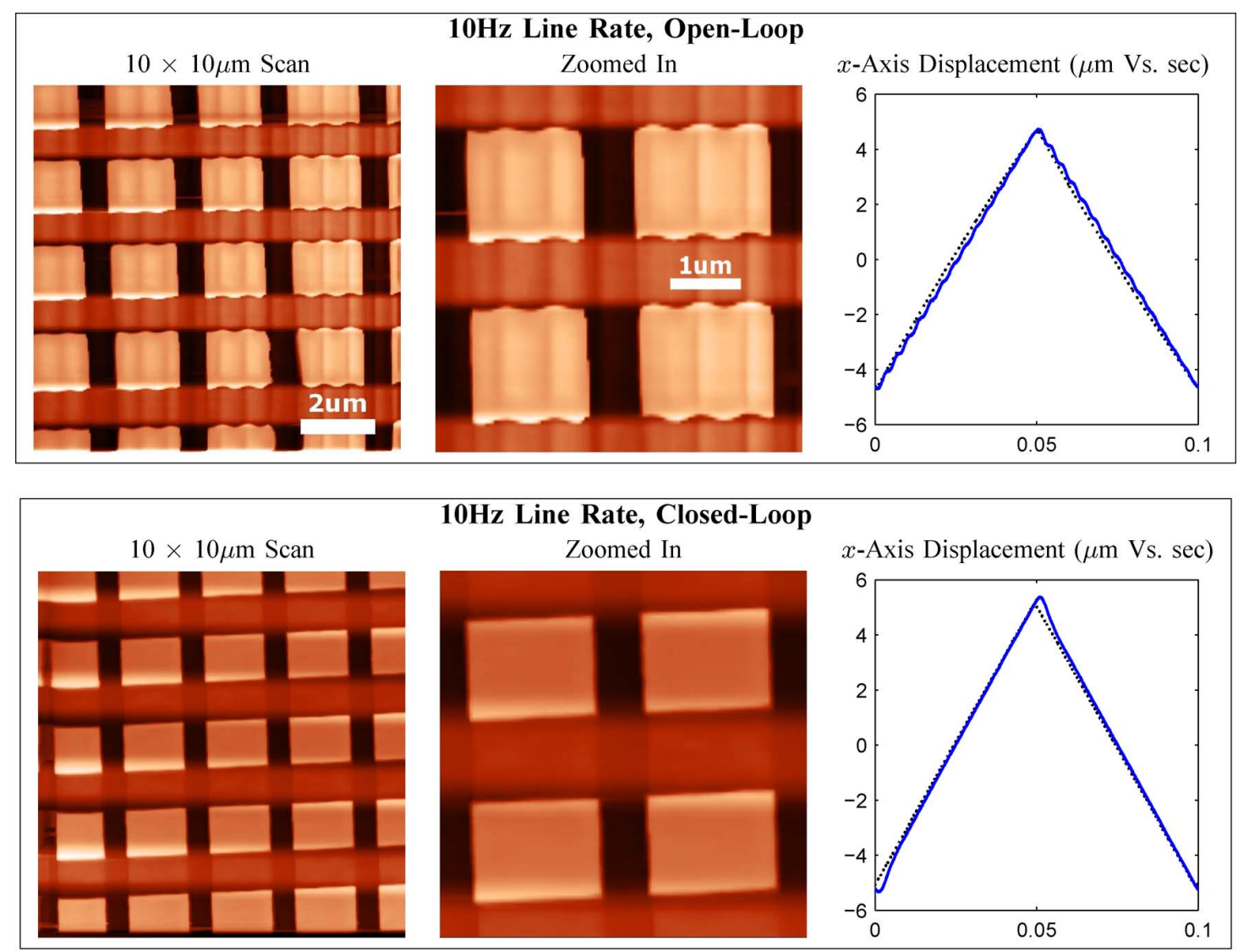

31Hz Line Rate, Open-Loop

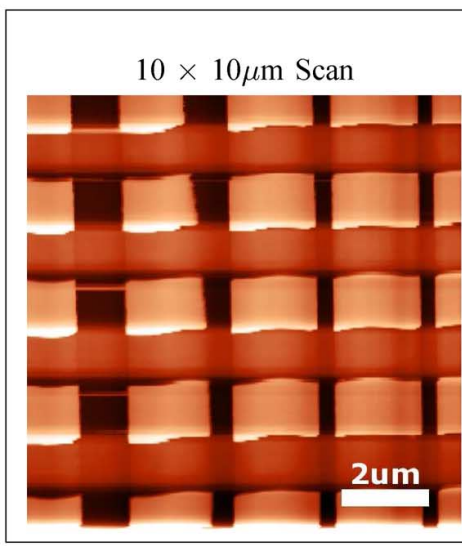

Zoomed In

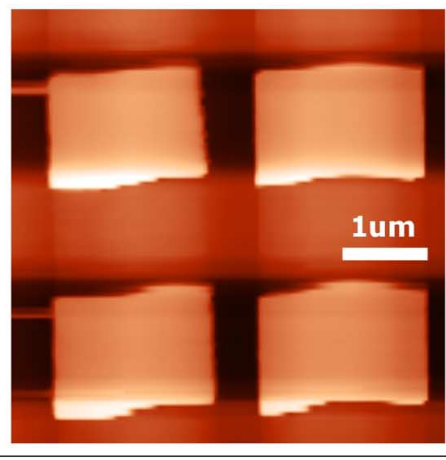

31Hz Line Rate, Closed-Loop

$10 \times 10 \mu \mathrm{m}$ Scan

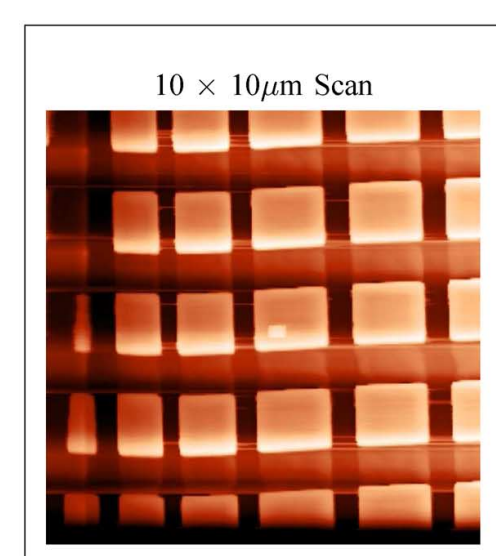

Zoomed In

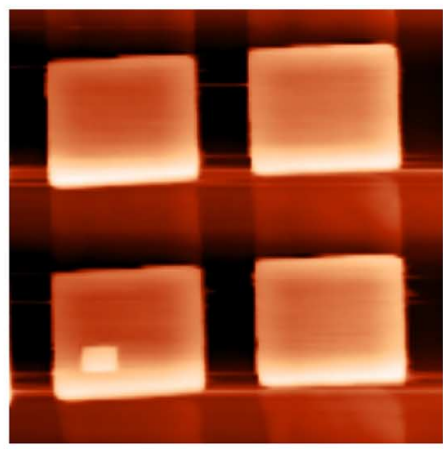

$x$-Axis Displacement ( $\mu \mathrm{m}$ Vs. sec)

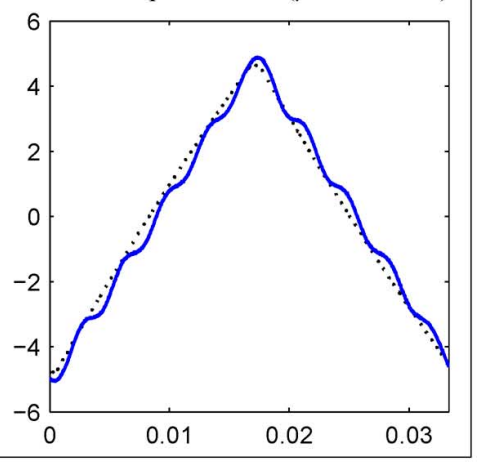

$x$-Axis Displacement ( $\mu \mathrm{m}$ Vs. sec)

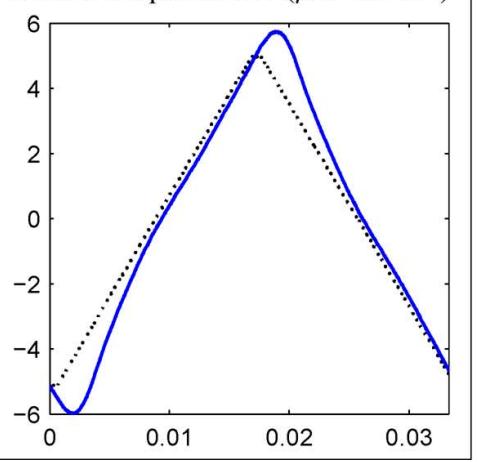

Fig. 9. Comparison of images recorded at 10 and $31 \mathrm{~Hz}$ with open- and closed-loop control of the sample scanner: (a) $10 \mathrm{~Hz}$ line rate (open-loop); (b) $10 \mathrm{~Hz}$ line rate (closed-loop); (c) $31 \mathrm{~Hz}$ line rate (open-loop); (d) $31 \mathrm{~Hz}$ line rate (closed-loop). 

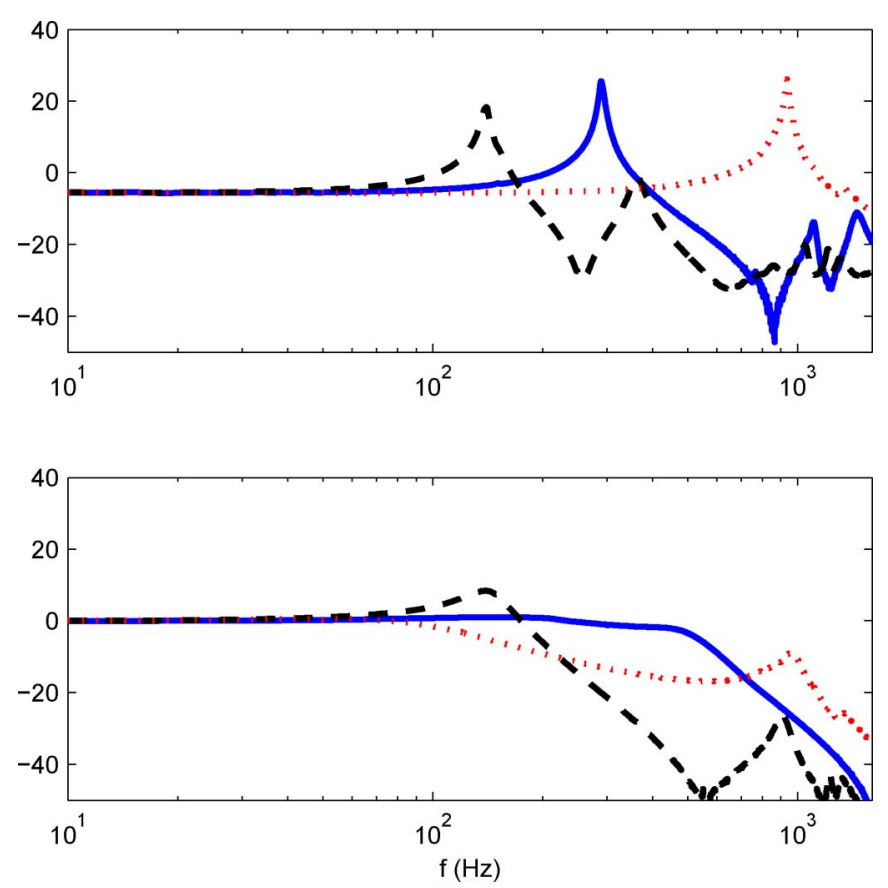

Fig. 10. Open-loop (top) and closed-loop (bottom) magnitude frequency response from the reference input voltage $r$ to measured sensor voltage $y$ (in decibel versus hertz). The three curves demonstrate the greatest range in frequency response that could occur in practice. The resonance frequencies range from the fully loaded case of $140 \mathrm{~Hz}$ (dashed line), to the nominal resonance frequency of $290 \mathrm{~Hz}$ (solid line), to the unloaded frequency of $934 \mathrm{~Hz}$ (dotted line).

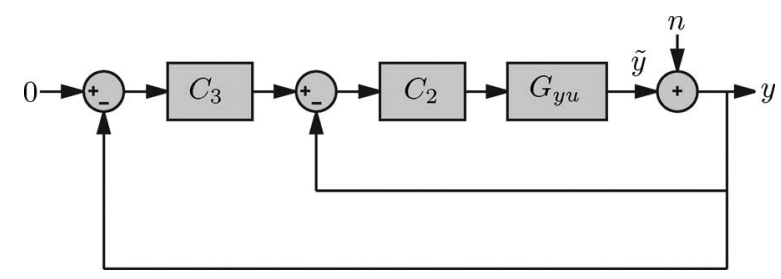

Fig. 11. Feedback diagram representing the effect of sensor noise $n$ on the true position $\tilde{y}$.

performance that decays gracefully as the resonance frequency drops. The main limitation to robustness is the integral tracking controller $C_{3}$. With decreasing resonance frequency, the phase margin of this controller slowly degrades, hence, it must be designed to tolerate the lowest expected resonance frequency. As the phase margin reduces, there is also some peaking introduced into the closed-loop tracking response, this can be observed for the lowest resonance frequency in Fig. 10.

\section{NOISE PENALTy}

A drawback of improved closed-loop bandwidth is increased sensor-induced noise. With a damping controller present, the feedback bandwidth is significantly increased. In this Section the damping controller's effect on sensor-induced position noise is examined.

To examine the system's noise performance, the measured position $y$ is split into the actual position $\tilde{y}$ and the additive

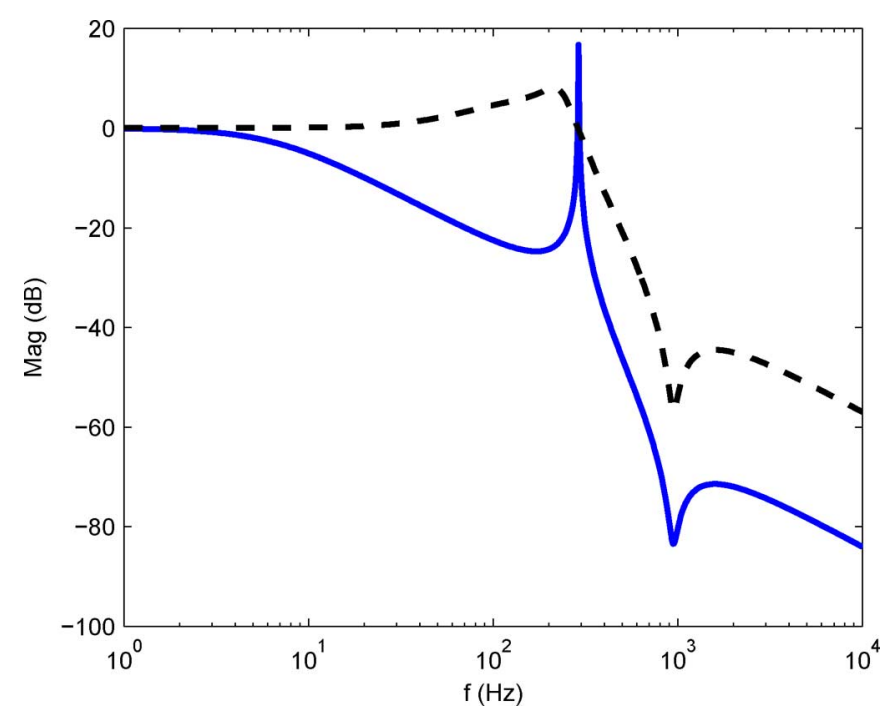

Fig. 12. Noise sensitivity $\tilde{y} / n$ of a slow integral controller (solid line) and controller with damping and fast integral action (dashed line).

(a)

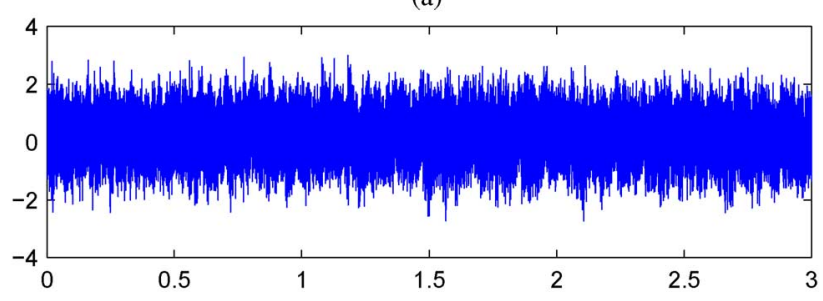

(b)

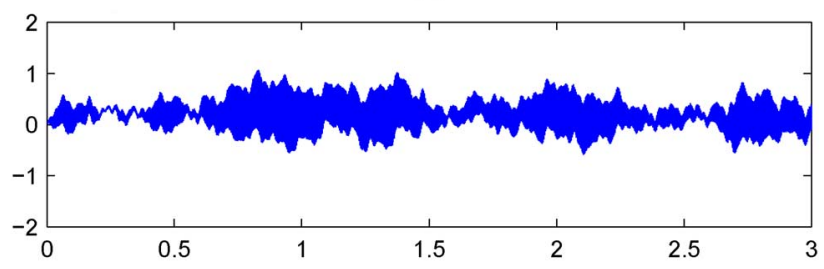

(c)

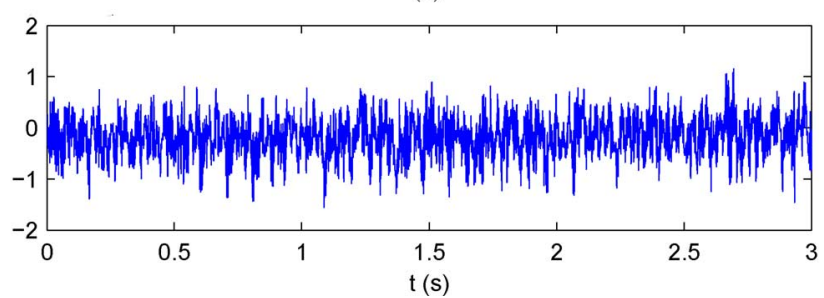

Fig. 13. (a) Measured sensor noise (in nanometers) and the resulting position noise of the integral controller (b) and integral controller with damping (c).

sensor noise $n$ as shown in Fig. 11. That is

$$
y=\tilde{y}+n .
$$

The transfer function from the sensor noise $n$ to the actual displacement $\tilde{y}$, referred to as the noise sensitivity transfer function, is

$$
\frac{\tilde{y}}{n}=\frac{-C_{2}\left(1+C_{3}\right) G_{y u}}{1+C_{2}\left(1+C_{3}\right) G_{y u}}
$$




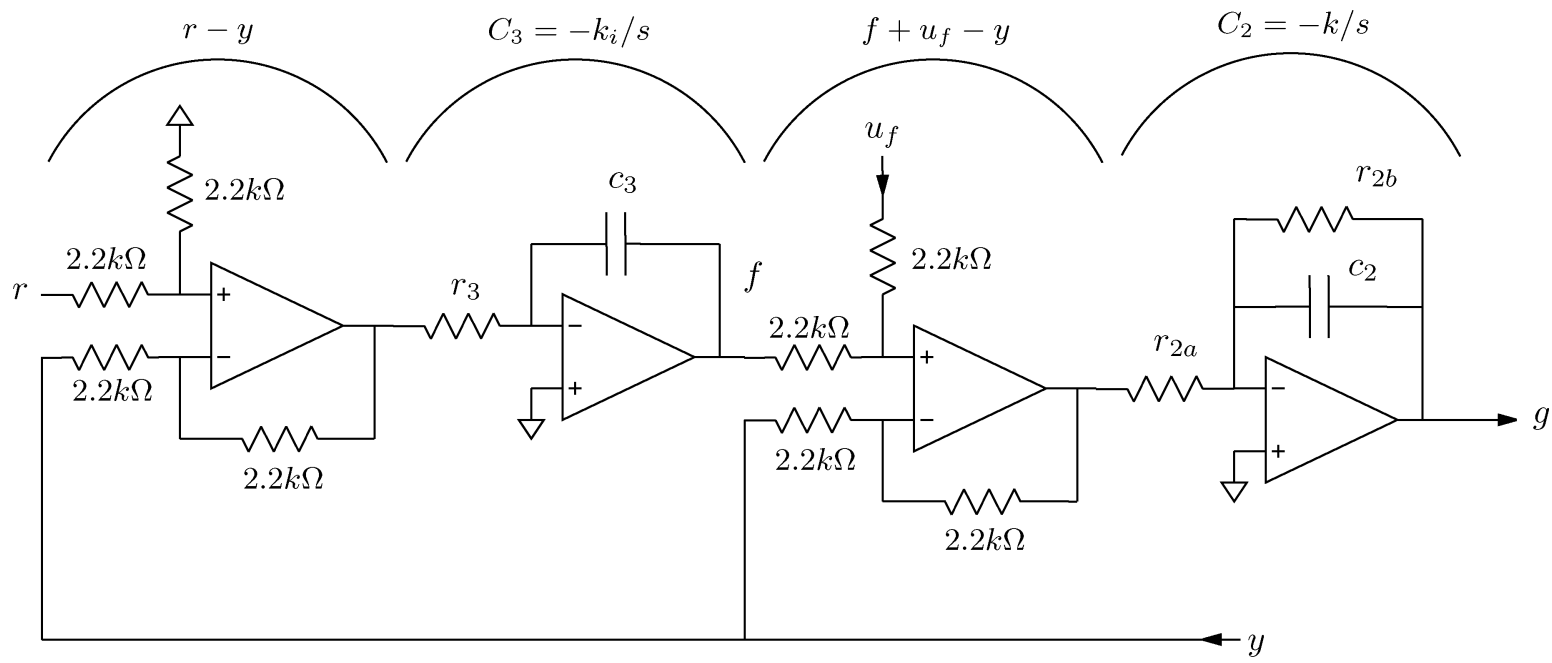

Fig. 14. Analog implementation of the IRC damping and tracking controller.

A useful observation is that if a damping controller is present, the noise sensitivity is not strongly affected by the tracking controller gain $C_{3}$. Thus, if a damping controller is employed, the tracking controller should be tuned to the highest practical gain as there is little noise penalty in doing so.

The most basic controller discussed in the previous section is a slow $\left(k_{i}=80\right)$ integral controller. With a bandwidth of only $15 \mathrm{~Hz}$, this is the control option with least noise. The noise sensitivity of the slow integral controller is plotted in Fig. 12. Also plotted is the noise sensitivity of the high-performance damping and tracking controller discussed in the previous Section. Although the noise sensitivity of the slow integral controller has a lower bandwidth, it also contains a lightly damped resonance which results in amplified sensor noise over a small bandwidth. In contrast, the damping and tracking controller has a wider bandwidth but no significant resonance.

To quantify the practical impact on positioning performance, both the noise sensitivity and noise density must be taken into account. By measuring the actual sensor noise, its effect on positioning noise can be simulated by filtering with the noise sensitivity (11). A three second record of the measured sensor noise and the resulting closed-loop position noise are plotted in Fig. 13. The rms noise values are also listed in Fig. 13. Clearly, with different controllers, the character of the noise is also quite different. While the slow integral controller contains low-frequency noise plus randomly excited resonance, the higher performance controller results in a more uniform spectrum but with a wider noise bandwidth. Considering that the closed-loop bandwidth has been increased from 15 to $490 \mathrm{~Hz}$, the increase in rms noise from 0.30 to $0.39 \mathrm{~nm}$ is negligible.

\section{ANALOG IMPLEMENTATION}

Due to the simplicity of the IRC damping and tracking controller, it is straight forward to implement in both analog and digital form. Although a digital implementation was used in previous sections, similar experiments using an analog controller produced identical results.
The IRC damping and tracking controller shown in Fig. 5 can be implemented directly with the analog circuit shown in Fig. 14. Although the controller requires only two operational amplifier (opamp), the four opamps circuit shown in Fig. 14 is easier to understand, trouble-shoot and tune (if necessary).

The operation of the circuit is self-explanatory. The first stage is a unity-gain differential amplifier that implements the subtraction function $r-y$. The second stage is an inverting integrator that implements the tracking controller $C_{3}=-k_{i} / s$. The corresponding circuit transfer function is $-1 / r_{3} c_{3} s$, which results in the equality $r_{3} c_{3}=1 / k_{i}$.

The third stage is a unity-gain differential amplifier with two noninverting inputs for $f$ and $u_{f}$. The final stage implements the IRC controller $C_{2}$, where

$$
C_{2}=\frac{-k}{s-k D_{f}} .
$$

The circuit transfer function is

$$
\frac{-\frac{1}{r_{2 a} c_{2}}}{s+\frac{1}{r_{2 b} c_{2}}} .
$$

As $k$ is positive and $D_{f}$ is negative, the equalities are

$$
r_{2 a} c_{2}=\frac{1}{k} \text { and } r_{2 b} c_{2}=\frac{1}{k D_{f}} .
$$

In both of the integrating stages, a $100 \mathrm{nF}$ polypropylene capacitor is recommended. The polypropylene dielectric is highly linear and temperature stable. These capacitors are also readily available with tolerances of $1 \%$. Other acceptable dielectric materials are polycarbonate and polyester. The capacitance value should not be less than $100 \mathrm{nF}$ to avoid large resistances that contribute thermal noise and amplify current noise. The opamps should have a gain-bandwidth product of around $10 \mathrm{MHz}$ or greater to avoid controller phase lag. The opamps should also be suited to a source impedance in the $\mathrm{k} \Omega$ range with the lowest possible noise corner frequency. The Texas Instruments OPA227, used in this paper, is a suitable device which is readily available at low cost. Another useful IC is the OPA4227 which contains 
four opamps and can implement the entire controller with one part.

The component values used to implement the controller parameters listed in Table I are

\begin{tabular}{|c|c|c|c|c|}
\hline$r_{3}$ & $c_{3}$ & $r_{2 a}$ & $r_{2 b}$ & $c_{2}$ \\
\hline \hline $25 \mathrm{k} \Omega$ & $100 \mathrm{nF}$ & $5.5 \mathrm{k} \Omega$ & $6.2 \mathrm{k} \Omega$ & $100 \mathrm{nF}$ \\
\hline
\end{tabular}

\section{CONCLUSION}

In this paper, IRC was applied to damp the first resonant mode of a scanning probe microscope positioning stage. Compared to a standard integral tracking controller, the IRC controller permitted an increase in closed-loop tracking bandwidth from 15 to $490 \mathrm{~Hz}$. The stability margins were simultaneously improved from 2.5 to $7 \mathrm{~dB}$ gain margin. Although the higher performance controller has a wider noise bandwidth, this bandwidth does not include the lightly damped resonance exhibited by standard tracking controllers. Consequently, the positioning noise was only increased from 0.30 to $0.39 \mathrm{~nm}$ rms. This is a negligible increase considering the large improvements in tracking bandwidth and image quality.

Besides the improved performance, other benefits of the proposed controller include ease of implementation and robustness. As the combined IRC and tracking controller is only second order, it is easily implemented with a simple analog circuit. The controller is also extremely robust to changes in resonance frequency.

Closed-loop stability and satisfactory performance was achieved in spite of a resonance frequency variation from 290 to $934 \mathrm{~Hz}$. Such large variations are commonly exhibited by piezoelectric tube scanners used with small samples and larger loads, for example, liquid cells and heating stages.

Experimental images using an NT-MDT microscope demonstrated a substantial improvement in image quality due to the elimination of scan-induced vibration.

\section{REFERENCES}

[1] G. Binnig and H. Rohrer, "The scanning tunneling microscope," Sci. Amer, vol. 253, pp. 50-56, 1986.

[2] S. M. Salapaka and M. V. Salapaka, "Scanning probe microscopy," IEEE Control Syst. Mag., vol. 28, no. 2, pp. 65-83, Apr. 2008.

[3] R. Koops and G. A. Sawatzky, "New scanning device for scanning tunneling microscope applications," Rev. Sci. Instrum., vol. 63, no. 8, pp. 40084009, Aug. 1992.

[4] T. Ando, N. Kodera, D. Maruyama, E. Takai, K. Saito, and A. Toda, "A high-speed atomic force microscope for studying biological macromolecules in action," Jpn. J. Appl. Phys., vol. 41, no. 7B, pp. 4851-4856, 2002.

[5] A. Sebastian and S. M. Salapaka, "Design methodologies for robust nanopositioning," IEEE Trans. Control Syst. Technol., vol. 13, no. 6, pp. 868876, Nov. 2005.

[6] G. Schitter, K. J. Åstrom, B. DeMartini, P. J. Thurner, K. L. Turner, and P. K. Hansma, "Design and modeling of a high-speed AFM-scanner," IEEE Trans. Control Syst. Technol., vol. 15, no. 5, pp. 906-915, Sep. 2007.

[7] K. K. Leang and A. J. Fleming, "High-speed serial-kinematic AFM scanner: Design and drive considerations," Asian J. Control, vol. 11, no. 2, pp. 144-153, Mar. 2009.

[8] S. O. R. Moheimani, "Accurate and fast nanopositioning with piezoelectric tube scanners: Emerging trends and future challenges,," Rev. Sci. Instrum., vol. 79, no. 7, pp. 071 101-1-071 101-11, Jul. 2008.
[9] G. Schitter and A. Stemmer, "Identification and open-loop tracking control of a piezoelectric tube scanner for high-speed scanning probe microscopy," IEEE Trans. Control Syst. Technol., vol. 12, no. 3, pp. 449-454, May 2004.

[10] J. Maess, A. J. Fleming, and F. Allgöwer, "Simulation of dynamicscoupling in piezoelectric tube scanners by reduced order finite element models," Rev. Sci. Instrum., vol. 79, pp. 015 105-1-015 105-9, Jan. 2008

[11] Piezoelectric Ceramics: Principles and Applications, APC International Ltd., Mackeyville, PA, 2006.

[12] A. J. Fleming and K. K. Leang, "Charge drives for scanning probe microscope positioning stages," Ultramicroscopy, vol. 108, no. 12, pp. 15511557, Nov. 2008.

[13] B. Yao, F.-S. Chien, S. Chen, P.-W. Lui, and G. Peng, "Scanning probe lithography with real time position control interferometer," in Proc. IEEENANO Conf., 2002, pp. 13-15.

[14] G. Yang, J. A. Gaines, and B. J. Nelson, "A supervisory wafer-level 3D microassembly system for hybrid MEMS fabrication," J. Intell. Robot. Syst., vol. 37, no. 1, pp. 42-68, 2003.

[15] M. J. Rost, L. Crama, P. Schakel, E. van Tol, G. B. E. M. van VelzenWilliams, C. F. Overgauw, H. ter Horst, H. Dekker, B. Okhuijsen, M. Seynen, A. Vijftigschild, P. Han, A. J. Katan, K. Schoots, R. Schumm, W. van Loo, T. H. Oosterkamp, and J. W. M. Frenken, "Scanning probe microscopes go video rate and beyond," Rev. Sci. Instrum., vol. 76, pp. $053710-1-053710-9,2005$.

[16] S. Thian, Y. Tang, J. Fuh, Y. Wong, L. Lu, and H. Loh, "Micro-rapidprototyping via multi-layered photo-lithography," Int. J. Adv. Manuf. Technol., vol. 29, no. 9-10, pp. 1026-1032, Jul. 2006.

[17] L. M. Picco, L. Bozec, A. Ulcinas, D. J. Engledew, M. Antognozzi, M. A. Horton, and M. J. Miles, "Breaking the speed limit with atomic force microscopy," Nanotechnology, vol. 18, pp. 044 030-1-044 030-4, 2007.

[18] D. Y. Abramovitch, S. Hoen, and R. Workman, "Semi-automatic tuning of PID gains for atomic force microscopes," in Proc. Amer. Control Conf., Seattle, WA, Jun. 2008, pp. 2684-2689.

[19] B. Bhikkaji, M. Ratnam, A. J. Fleming, and S. O. R. Moheimani, "Highperformance control of piezoelectric tube scanners," IEEE Trans. Control Syst. Technol., vol. 5, no. 5, pp. 853-866, Sep. 2007.

[20] B. Bhikkaji, M. Ratnam, and S. O. R. Moheimani, "PVPF control of piezoelectric tube scanners.," Sens. Actuators: A Phys., vol. 135, no. 2, pp. 700-712, Apr. 2007.

[21] A. J. Fleming and S. O. R. Moheimani, "Sensorless vibration suppression and scan compensation for piezoelectric tube nanopositioners," IEEE Trans. Control Syst. Technol., vol. 14, no. 1, pp. 33-44, Jan. 2006.

[22] S. S. Aphale, A. J. Fleming, and S. O. R. Moheimani, "High speed nanoscale positioning using a piezoelectric tube actuator with active shunt control," Micro Nano Lett., vol. 2, no. 1, no. 1, pp. 9-12, 2007.

[23] N. Kodera, H. Yamashita, and T. Ando, "Active damping of the scanner for high-speed atomic force microscopy," Rev. Sci. Instrum., vol. 76, no. 5, pp. 053 708-1-053 708-5, 2005.

[24] A. J. Fleming, A. G. Wills, and S. O. R. Moheimani, "Sensor fusion for improved control of piezoelectric tube scanners," IEEE Trans. Control Syst. Technol., vol. 15, no. 6, pp. 1265-6536, Nov. 2008.

[25] S. Devasia, E. Eleftheriou, and S. O. R. Moheimani, "A survey of control issues in nanopositioning," IEEE Trans. Control Syst. Technol., vol. 15, no. 5, pp. 802-823, Sep. 2007.

[26] J. A. Butterworth, L. Y. Pao, and D. Y. Abramovitch, "A comparison of control architectures for atomic force microscopes," Asian J. Control, vol. 11, no. 2, pp. 175-181, Mar. 2009.

[27] S. Devasia, "Should model-based inverse inputs be used as feedforward under plant uncertainty?," IEEE Trans. Autom. Control, vol. 47, no. 11, pp. 1865-1871, Nov. 2002.

[28] Y. Wu and Q. Zou, "Iterative control approach to compensate for both the hysteresis and the dynamics effects of piezo actuators," IEEE Trans. Control Syst. Technol., vol. 15, no. 5, pp. 936-944, Sep. 2007.

[29] K. Kim and Q. Zou, "Model-less inversion-based iterative control for output tracking: Piezo actuator example," in Proc. Amer. Control Conf., Seattle, WA, Jun. 2008, pp. 2710-2715.

[30] Y. Li and J. Bechhoefer, "Feedforward control of a piezoelectric flexure stage for AFM," in Proc. Amer. Control Conf., Seattle, WA, Jun. 2008, pp. 2703-2709.

[31] S. S. Aphale, A. J. Fleming, and S. O. R. Moheimani, "Integral resonant control of collocated smart structures," Smart Mater. Struct., vol. 16, pp. 439-446, 2007.

[32] B. Bhikkaji and S. O. R. Moheimani, "Integral resonant control of a piezoelectric tube actuator for fast nanoscale positioning," IEEE/ASME Trans. Mechatronics, vol. 13, no. 5, pp. 530-537, Oct. 2008. 
[33] T. McKelvey, H. Akcay, and L. Ljung, "Subspace based multivariable system identification from frequency response data," IEEE Trans. Autom. Control, vol. 41, no. 7, pp. 960-978, Jul. 1996.

[34] K. K. Leang and S. Devasia, "Feedback-linearized inverse feedforward for creep, hysteresis, and vibration compensation in AFM piezoactuators," IEEE Trans. Control Syst. Technol., vol. 15, no. 5, pp. 927-935, Sep. 2007.

[35] S. Devasia, "Review of feedforward approaches for nano precision positioning in high speed SPM operation," in Proc. IFAC World Congr., Seoul, Korea, Jul. 2008, pp. 9221-9229.

[36] A. J. Fleming and A. G. Wills, "Optimal periodic trajectories for bandlimited systems," IEEE Trans. Control Syst. Technol., vol. 13, no. 3, pp. 552-562, May 2009.

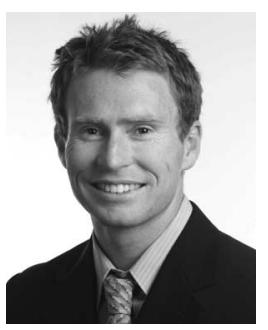

Andrew J. Fleming (S'02-M'03) was born in Dingwall, Scotland, in 1977. He received the Bachelor of Electrical Engineering degree and the Ph.D degree from the University of Newcastle, Callaghan, Australia, in 2000 and 2004, respectively.

$\mathrm{He}$ is currently an Australian Research Council Fellow with the School of Electrical Engineering and Computer Science, University of Newcastle. $\mathrm{He}$ is also the Deputy Program Leader for Mechatronics at the Center of Excellence for Complex Dynamic Systems and Control, University of Newcastle. His research interests include nanopositioning, high-speed scanning probe microscopy, microcantilever sensors, and sensor-less control of sound and vibration. He is the coauthor of two books, several patent applications, and more than 80 Journal and Conference papers.

Dr. Fleming was awarded the University of Newcastle Vice-Chancellors Award for Researcher of the Year and the Faculty of Engineering and Built Environment Award for Research Excellence, in 2007. He was also the recipient of the IEEE Control Systems Society Outstanding Paper Award for research published in the IEEE TRANSACTIONS ON CONTROL SYSTEMS TECHNOLOGY, in 2007. He is the Associate Editor of Advances in Acoustics and Vibration.

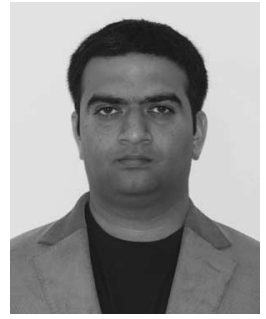

Sumeet S. Aphale (M'09) received the Bachelors degree in electrical engineering from the University of Pune, Pune, India, in 1999, and the M.S. and Ph.D. degrees in electrical engineering from the University of Wyoming, Laramie, WY, in 2003 and 2005, respectively.

From October 2005 to June 2008, he was a Member of the Center of Excellence for Complex Dynamic Systems and Control, University of Newcastle, Callaghan, Australia. He was a Senior Research Fellow with the Center for Applied Dynamics Research, University of Aberdeen, Aberdeen, U.K. He is currently a Lecturer with the School of Engineering, University of Aberdeen. His research interests include robot kinematics and control, vibration control applications as well as design and control of nanopositioning systems for applications such as scanning probe microscopy.

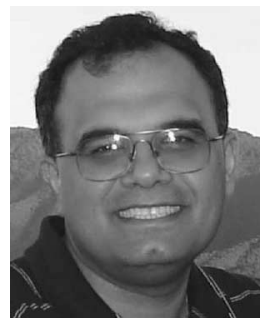

S. O. Reza Moheimani (SM'00) received the Ph.D. degree in electrical engineering from the Australian Defence Force Academy, University of New South Wales, Canberra, A.C.T., Australia, in 1996.

$\mathrm{He}$ is currently a Professor in the School of Electrical Engineering and Computer Science, University of Newcastle, Callaghan, N.S.W., where he holds an Australian Research Council Future Fellowship. He is the Associate Director of the Australian Research Council Center for Complex Dynamic Systems and Control, an Australian Government Center of Excellence. He has held several visiting appointments at IBM Zurich Research Laboratory, Zurich, Switzerland. He is the author of two books, several edited volumes, and more than 200 refereed articles in archival journals and conference proceedings. His current research interests include applications of control and estimation in nanoscale positioning systems for scanning probe microscopy, and control of electrostatic microactuators in microelectromechanical systems, and data storage systems.

Dr. Moheimani is a Fellow of the Institute of Physics, London, U.K. He is the recipient of the 2007 IEEE TRANSACTIONS ON CONTROL SYSTEMS TECHNOLOGY outstanding Paper Award. He has served on the Editorial Board of a number of journals including the IEEE TRANSACTIONS ON CONTROL SYSTEMS TECHNOLOGY, and chaired several international conferences and workshops. 